INTERVENTIONAL CARDIOLOGY AND SURGERY

\title{
Coronary blood flow, metabolism, and function in dysfunctional viable myocardium before and early after surgical revascularisation
}

\author{
F Alamanni, A Parolari, A Repossini, E Doria, F Bortone, J Campolo, M Pepi, E Sisillo, M Naliato, \\ R Bigi, P Biglioli, O Parodi
}

Heart 2004;90:1291-1298. doi: 10.1136/hrt.2003.022327

See end of article for authors' affiliations

Correspondence to: Professor Oberdan Parodi, CNR Clinical Physiology Institute, Section of Milan, Niguarda $\mathrm{Ca}^{\prime}$ Granda Hospital, Piazza Ospedale Maggiore, 3-20162 Milan, Italy; ifcnig@tin.it

Accepted 12 March 2004
Objectives: To assess the link between perfusion, metabolism, and function in viable myocardium before and early after surgical revascularisation.

Design: Myocardial blood flow (MBF, thermodilution technique), metabolism (lactate, glucose, and free fatty acid extraction and fluxes), and function (transoesophageal echocardiography) were assessed in patients with critical stenosis of the left anterior descending coronary artery (LAD) before and 30 minutes after surgical revascularisation.

Setting: Tertiary cardiac centre.

Patients: 23 patients (mean (SEM) age 57 (1.7) years with LAD stenosis: 17 had dysfunctional viable myocardium in the LAD territory, as shown by thallium-201 rest redistribution and dobutamine stress echocardiography (group 1), and six had normally contracting myocardium (group 2).

Results: LAD MBF was lower in group 1 than in group $2(58(7) \vee 113(21) \mathrm{ml} / \mathrm{min}, \mathrm{p}<0.001)$ before revascularisation and improved postoperatively in group 1 (129 (133) $\mathrm{ml} / \mathrm{min}, \mathrm{p}<0.001$ ) but not in group $2(105(20) \mathrm{ml} / \mathrm{min}, p=0.26)$. Group 1 also had functional improvement in the LAD territory at intraoperative echocardiography (mean regional wall motion score from $2.6(0.85)$ to $1.5(0.98)$, $p<0.01$ ). Oxidative metabolism, with lactate and free fatty acid extraction, was found preoperatively and postoperatively in both groups; however, lactate and free fatty acid uptake increased after revascularisation only in group 1 .

Conclusions: MBF is reduced and oxidative metabolism is preserved at rest in dysfunctional but viable myocardium. Surgical revascularisation yields immediate perfusion and functional improvement, and increases the uptake of lactate and free fatty acids.
M yocardial hibernation refers to chronic cardiac dysfunction subtending critical coronary artery stenosis, which can be partially or completely restored by coronary revascularisation. ${ }^{1}$ Although metabolic adaptation in the myocardial areas supplied by critical stenotic lesions has been recognised, ${ }^{2}{ }^{3}$ other reports have questioned this hypothesis on the grounds of the possibility of cell dedifferentiation, ${ }^{4}$ cell degeneration, or both. ${ }^{5}$ The mechanism of chronic post-ischaemic reversible dysfunction is likely to be multifactorial and to entail ultrastructural and metabolic changes; the different contributions of each of these mechanisms may explain the variable extent and delay of functional recovery after successful revascularisation. However, a few clinical studies ${ }^{67}$ have addressed the hypothesis that the reversibility of chronic dysfunction after blood flow restoration provides evidence of a pathophysiological link between flow and function.

In the clinical setting, it is not known whether metabolic adaptation to chronic hypoperfusion is sustained by anaerobic metabolism that reverts after flow restoration or whether the physiological aerobic pathways are downregulated.

The aim of this study was to define preoperatively the behaviour of regional perfusion, metabolism, and function in viable myocardial regions with chronic asynergy and to assess the effects of coronary revascularisation on these parameters. To this purpose, we selected candidates for coronary artery bypass graft $(\mathrm{CABG})$ surgery with stable angina pectoris, critical coronary lesion in the left anterior descending coronary artery (LAD), and LAD dependent dysfunctional viable myocardium.

\section{METHODS}

\section{Study population}

One hundred and seven consecutive patients with stable angina pectoris and regional left ventricular dysfunction who underwent elective CABG surgery were screened for myocardial viability in the LAD territory according to the American College of Cardiology/American Heart Association guidelines. Twenty three patients (mean (SEM) age 57 (1.7) years) fulfilled the following inclusion criteria: (1) no history of previous myocardial infarction; (2) no unstable angina before surgery; (3) onset of clinical symptoms lasting no longer than one year; (4) evidence of critical ( $\geqslant 70 \%$ ) LAD stenosis and the possibility of achieving complete surgical revascularisation of all diseased vessels; and (5) no significant concomitant valvar disease, severe pulmonary disease, diabetes, or arterial hypertension. Seventeen patients had dysfunctional viable myocardium only in the LAD dependent territory as assessed by dobutamine stress echocardiography and rest redistribution thallium-201 single photon emission computed tomography $\left({ }^{201} \mathrm{Tl}\right.$-SPECT $)$ (group 1). The other six patients had normal left ventricular function (group 2). No patient among those with dysfunctional myocardium had a left ventricular aneurysm. Ten patients had single LAD disease, 10 patients had double

Abbreviations: $C A B G$, coronary artery bypass graft; $L A D$, left anterior descending coronary artery; MBF, myocardial blood flow; RPP, ratepressure product; ${ }^{201} \mathrm{Tl}-\mathrm{SPECT}$, thallium-201 single photon emission computed tomography 


\begin{tabular}{|c|c|c|c|}
\hline Variable & Group $1(n=17)$ & Group $2(n=6)$ & $\mathrm{p}$ Value \\
\hline Age (years) & $58(2)$ & $55(4)$ & 0.487 \\
\hline BSA $\left(\mathrm{m}^{2}\right)$ & $1.88(0.05)$ & $1.85(0.22)$ & 0.678 \\
\hline Male sex & 17 & 5 & 0.543 \\
\hline Previous $\mathrm{Ml}$ & 0 & 0 & 1 \\
\hline Stable angina & 17 & 6 & 1 \\
\hline Angiographic EF (\%) & $51.4(6.1)$ & $63.2(5.5)$ & $<0.001$ \\
\hline LAD lumen reduction (\%) & $92.4(9.5)$ & $89.8(10.9)$ & 0.585 \\
\hline RCA stenosis & 7 & 2 & \\
\hline Circumflex artery stenosis & 5 & 2 & \\
\hline Distal anastomoses & $2.5(0.2)$ & $1.9(0.3)$ & 0.081 \\
\hline LIMA use & 17 & 6 & 1 \\
\hline CPB time (min) & $73(7)$ & $59(8)$ & 0.311 \\
\hline Aortic cross clamp time (min) & $52(5)$ & $38(2)$ & 0.150 \\
\hline
\end{tabular}

vessel disease, and three patients had triple vessel disease. Table 1 shows the preoperative clinical and angiographic features and the operative findings in the two groups.

\section{Study protocol}

The baseline studies before revascularisation were two dimensional transthoracic echocardiography at rest for both groups, and dobutamine stress echocardiography and rest four hour redistribution ${ }^{201} \mathrm{Tl}$-SPECT for group 1 only. Dobutamine stress echocardiography was performed 3 (1.1) days before surgery and within one day of ${ }^{201} \mathrm{Tl}$-SPECT. Significant coronary artery disease was defined as the percentage reduction of the internal lumen diameter $\geqslant 70 \%$. Left ventricular function was evaluated in all of the patients by means of monoplane contrast ventriculography $\left(30^{\circ}\right.$ right oblique anterior view); left ventricular ejection fraction was calculated by the area-length method.

All of the patients were then studied during CABG surgery after opening of the pericardium, systemic heparinisation, and cannulation of the ascending aorta. Intraoperative studies were transoesophageal echocardiography, myocardial blood flow (MBF) measurement, and arterial and great cardiac vein blood sampling.

After the coronary bypass procedure was completed and about 30 minutes after the patient was weaned from cardiopulmonary bypass, transoesophageal echocardiography, MBF, and arterial and great cardiac vein blood sampling were repeated under the haemodynamic conditions closest to the preoperative ones (fig l).

All of the patients underwent transthoracic echocardiography at rest one week and three months after surgery.
The study protocol was approved by the ethics committee of the Centro Cardiologico Monzino. Written informed consent was obtained from all patients.

\section{Echocardiographic studies}

All patients underwent transthoracic echocardiography with a commercial ultrasonograph (Hewlett Packard Sonos 1500) and 2.5-3.5 MHz transducers. Standard parasternal short axis, apical four chamber, and apical two chamber views were acquired and recorded on VHS tape, and the baseline left ventricular ejection fraction was calculated. ${ }^{8}$ Subsequently, group 1 patients underwent dobutamine infusion and echocardiographic monitoring according to previously described methods. ${ }^{9}$ Data were analysed off line by two independent observers unaware of the clinical, scintigraphic, and angiographic findings; discrepancies were resolved by consensus.

Regional wall motion scores were assessed according to the 16 segment model from review of the videotapes in a quad screen format. The number of resting asynergic segments to the LAD territory, their resting motion score, and their maximum score improvement during dobutamine infusion were recorded. The average baseline and maximum improvement scores were computed. Contractile reserve was considered to be present either when the systolic wall thickening score improved in at least two adjacent abnormal segments or when subsequent doses were associated with an ischaemia induced worsening (biphasic response). Intraobserver and interobserver variabilities in describing improved or unchanged segments during dobutamine infusion in our laboratory are $4.5 \%$ and $9.2 \%$, respectively.

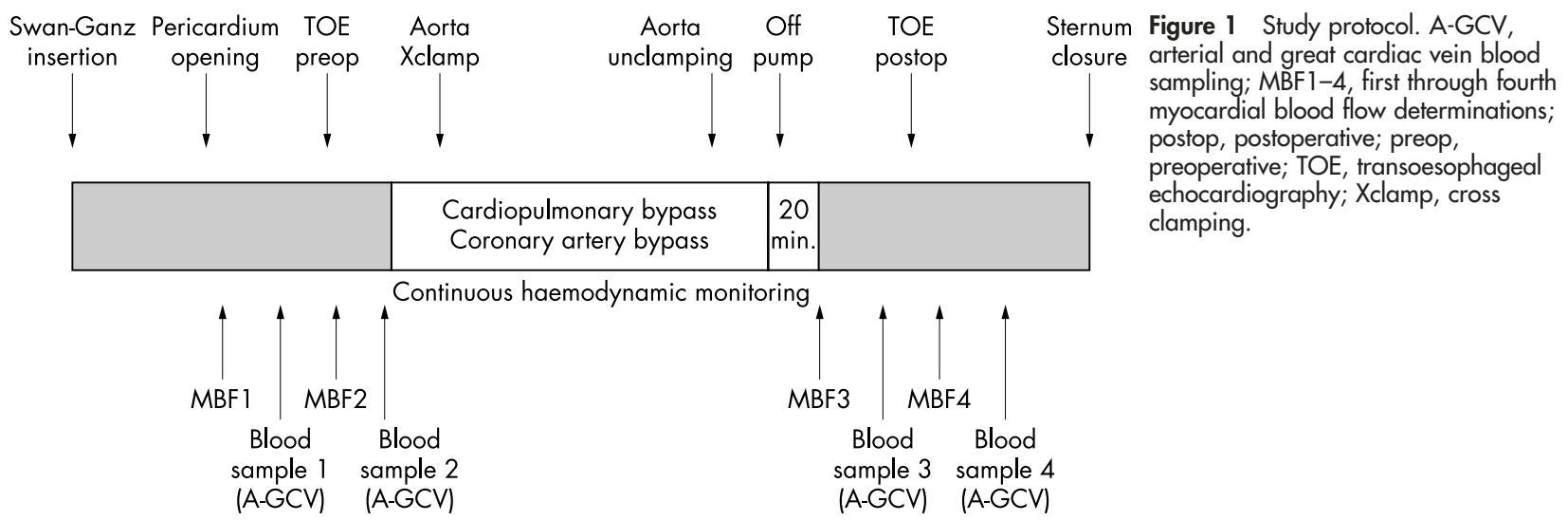


Intraoperative transoesophageal echocardiography was performed with a multiplane transoesophageal instrument (omniplane model 21364A, Hewlett Packard) with a $5 \mathrm{MHz}$ phased array transducer. The short axis, four chamber, and two chamber views were recorded with the transducer positioned in the mid oesophagus. Segmental wall motion was analysed by dividing the ventricle into the same 16 segments as those used for dobutamine stress echocardiography. A longitudinal image of the left ventricle orthogonal to the short axis, including the apex, and of the inferior and anterosuperior walls of the ventricle, allowed adequate visualisation of the endocardial surfaces.

\section{Rest redistribution ${ }^{201} \mathrm{TI}-\mathrm{SPECT}$}

Group l patients underwent rest $(2.8 \mathrm{mCi}, 103.6 \mathrm{MBq})$ four hour redistribution ${ }^{201}$ Tl-SPECT protocol within one day of dobutamine stress echocardiography. The tomograms $(64 \times 64$ matrix $)$ were acquired early and four hours after tracer injection with a rotating gamma camera (Starcam 3000, General Electric) and a low energy general purpose collimator in a $180^{\circ}$ circular orbit (from the $45^{\circ}$ right anterior oblique projection to the $45^{\circ}$ left posterior oblique projection) with 32 steps for 40 second acquisition. The left ventricular short axis slices were reconstructed every $6 \mathrm{~mm}$. From three representative apical, mid ventricular, and basal short axis sections, 16 segments matching the transoesophageal echocardiographic and transthoracic echocardiographic segments were automatically drawn by computer and analysed quantitatively.

The quantitative assessment was made by normalising the ${ }^{201} \mathrm{Tl}$ uptake in each segment to the segment with the highest uptake: ${ }^{201} \mathrm{Tl}$ activity was considered normal when it was $75-$ $100 \%$, moderately reduced at $50-74 \%$, and severely reduced at $0-49 \%$. A segment was considered viable when the ${ }^{201} \mathrm{Tl}$ activity was $\geqslant 50 \%$ of the maximum activity recorded in the resting or redistribution images.

\section{Surgical procedure}

The management of the patients during and after the operation has been described elsewhere. ${ }^{10}$

Blood pressures were monitored with the use of a radial artery and a flow directed pulmonary artery catheter. The patients were cooled to $29-31^{\circ} \mathrm{C}$. For purposes of myocardial protection, the patients received multidose antegrade and retrograde high potassium cold crystalloid cardioplegia. Distal graft anastomoses were positioned during aortic cross clamping: the LAD and its branches were always revascularised with the left in situ internal mammary artery, with sequential anastomoses as needed. When necessary, additional coronary vessels were revascularised with the great saphenous vein. Complete revascularisation was achieved in every patient.

\section{Haemodynamic measurements}

Heart rate, systolic, diastolic, and mean aortic pressures, right atrial pressure, pulmonary capillary wedge pressure, and cardiac output (by the thermodilution technique) were measured. The derived haemodynamic measurements were systemic vascular resistance and rate-pressure product (RPP).

All of the measurements were recorded in duplicate in the operating room during flow and echocardiographic measurements.

\section{Coronary blood flow measurement}

Soon after cannulation of the ascending aorta, a thermodilution catheter (CCS 7W 9A Ball End, Webster Laboratories) connected to a flowmeter (CF-300A, Webster Laboratories) was surgically advanced into the coronary sinus and inserted into the great cardiac vein under direct vision. Advancement of the thermodilution catheter tip to where the anterior intraventricular vein meets the great cardiac vein provides the most stable position and the most selective measurement of LAD territory outflow. The constant position of the catheter tip, critical to assure the reliability of measurement, was checked by direct vision, with the position of the tip marked by a metal clip applied to the fat behind the left atrial appendage. Measurements at this position indicate primarily LAD venous outflow.

Under stable haemodynamic conditions, MBF was measured with a syringe infuser to inject room temperature normal saline at a constant rate $(50 \mathrm{ml} / \mathrm{min})$ through the thermodilution catheter. The deflection from baseline was recorded with a jet polygraph (Mingograf 34, Siemens Elema) and millimetre paper, and compared with a standard deflection. The great cardiac vein flow measurements were considered stable when there was a $<10 \%$ variation in the resting value obtained from three repeated measurements. The measurements were made in duplicate using the continuous infusion thermodilution method. ${ }^{11}$ Because basal MBF is closely related to the RPP, MBF data before and after revascularisation in both patient groups are reported after correction for RPP by the equation RPP corrected $\mathrm{MBF}=$ basal $\mathrm{MBF} \times($ mean patient $\mathrm{RPP} /$ individual $\mathrm{RPP})$, as previously described. ${ }^{12}$

\section{Arterial and great cardiac vein blood sampling}

Before and after the MBF determinations, blood was simultaneously collected from the radial artery and great cardiac vein catheter in two syringes (one heparinised and one containing fluoride-EDTA as anticoagulant) and immediately capped and put in ice. The contents of the heparinised syringe were used for blood gas analyses. Myocardial oxygen consumption was calculated as the product of great cardiac vein flow and the artero-great cardiac vein blood oxygen concentration difference and corrected for RPP. ${ }^{12}$ The second syringe was immediately centrifuged in a refrigerated centrifuge to separate the plasma and metabolically determine the plasma concentrations of lactate, glucose, and free fatty acids by means of modified versions of previously described methods. ${ }^{13}{ }^{14}$ Lactate, glucose, and free fatty acid concentrations in each plasma sample (two before and two after CABG surgery) were measured in duplicate and the mean values were used for analysis.

\section{Postoperative echocardiography and follow up}

One week and three months after revascularisation, all of the patients underwent a transthoracic echocardiography examination. Results were reviewed by two independent observers to assess segmental wall motion.

\section{Statistical analysis}

The data are presented as mean (SEM). Student's $t$ test for paired and unpaired samples or one way analysis of variance (followed by Bonferroni correction when indicated) was used for within group or between groups comparisons of continuous variables. Fisher's exact test was used to compare categorical variables. The analysis of Bland and Altman ${ }^{15}$ was used to assess the agreement between repeated measurements of MBF before and after revascularisation. A probability value of $\mathrm{p}<0.05$ was considered significant. SPSS version 6.0 for Windows (SPSS Inc, Chicago, Illinois, USA) was used for all analyses.

\section{RESULTS}

\section{Clinical features}

The patients in group 1 were found to have 78 asynergic LAD related segments (4.6 per patient) at both preoperative 
transthoracic echocardiography and intraoperative preprocedure transoesophageal echocardiography. Hypokinesis, akinesis, or dyskinesis was found in 24,47 , and 7 segments, respectively. Seventy one of these segments (91\%) were viable on ${ }^{201} \mathrm{Tl}$-SPECT and $61(78 \%)$ on dobutamine stress echocardiography. Only 10 segments (five patients) viable on ${ }^{201}$ Tl-SPECT were judged to be non-viable by stress echocardiography criteria. All of these segments were akinetic at baseline evaluation. Seven of 10 segments had improved at three months' follow up. Only seven segments (9\%) were judged to be necrotic by both ${ }^{201}$ Tl-SPECT and stress echocardiography.

All of the CABG operations were successful and uncomplicated and no patient needed inotropic drugs.

\section{Haemodynamic measurements}

Table 2 shows the haemodynamic profiles of the patients in groups 1 and 2 before and after CABG surgery. In group 1 , pulmonary capillary wedge pressure did not change; heart rate, cardiac output, and RPP increased significantly; and peripheral vascular resistance decreased. In group 2, there was no change in right atrial and pulmonary capillary wedge pressure, cardiac output, or RPP; heart rate increased significantly; and both systolic and diastolic arterial blood pressures decreased significantly. The reduction in peripheral vascular resistance, albeit non-significant, in group 2 was similar to that observed in group 1 .

\section{Myocardial blood flow}

LAD blood flow was significantly lower in group 1 than in group 2 before revascularisation. After surgery, RPP corrected MBF increased significantly in group 1, whereas no significant changes were detected in group 2. Moreover, there were no significant differences between repeated measurements in both groups and in postoperative flow measurements between groups (table 3, fig 2), as documented by Bland-Altman analysis of agreement, which showed a mean MBF change between duplicate measurements of $1.5 \mathrm{ml} / \mathrm{min}$ and $1.7 \mathrm{ml} / \mathrm{min}$ before and after revascularisation, respectively. Limits of agreement ranged from -12.5 to $15.6 \mathrm{ml} / \mathrm{min}$ in pre-revascularisation measurements and from -14.2 to $19.3 \mathrm{ml} / \mathrm{min}$ in post-revascularisation measurements (mean (2SD)).

The postoperative increase in group $1 \mathrm{MBF}$ was associated with a significant increase in myocardial oxygen consumption. In group 2 there were no significant changes (table 3 ). Lastly, no significant changes in myocardial oxygen extraction were detected perioperatively in both groups or between groups (table 3 ).

\section{Metabolic determinations}

In both groups, there was no significant difference between the prerevascularisation and post-revascularisation lactate (fig 3), glucose (fig 4), and free fatty acid extraction rates (fig 5); lactate (fig 3A) and free fatty acid fluxes (fig 5A) increased significantly in group 1 after surgery. No significant differences were detected in preoperative and postoperative extraction rates and fluxes between group 1 and 2, with the exception of preoperative free fatty acid fluxes, which were significantly higher in group 2 patients $(p=0.039)$.

\section{Left ventricular function}

No significant differences were observed between wall motion scores taken during baseline transthoracic echocardiography at rest and those during prerevascularisation transoesophageal echocardiography. Regional wall motion improved significantly at dobutamine stress echocardiography with respect to baseline transthoracic echocardiography and preoperative transoesophageal echocardiography. The post-revascularisation regional wall motion score at transoesophageal echocardiography improved significantly with respect to pre-revascularisation measurements and it was of the same magnitude as that observed at transthoracic echocardiography eight days and three months after surgery (table 4). Among the 78 asynergic segments, 89\%, 83\%, and $43 \%$ of the hypokinetic, akinetic, and dyskinetic segments, respectively, improved or normalised function. Figure 6 shows the individual behaviour of perioperative great cardiac vein blood flow and regional wall motion score changes in group l patients.

No perioperative functional changes in the LAD wall segments were observed in group 2; furthermore, the segments unrelated to the LAD territory displayed neither a hyperkinetic pattern nor the occurrence of new regional wall motion abnormalities after revascularisation.

\section{DISCUSSION}

This is the first report of a simultaneous assessment of regional $\mathrm{MBF}$, metabolism, and function in patients with stable angina pectoris and dysfunctional viable myocardium before and soon after surgical coronary revascularisation.

The main findings of this study are that, firstly, viable myocardial regions with chronic asynergy have a reduced baseline MBF, which increases soon after revascularisation; secondly, improvement of perfusion is paralleled by an early recovery of contractile function in most of the asynergic territories; and thirdly, oxidative metabolism is present in these regions and increases substantially after blood flow is restored.

Table 2 Haemodynamic data before and after coronary artery bypass graft surgery

\begin{tabular}{|c|c|c|c|c|c|c|c|c|}
\hline & \multicolumn{3}{|c|}{ Group 1 patients $(n=17)$} & \multicolumn{3}{|c|}{ Group 2 patients $(n=6)$} & \multicolumn{2}{|c|}{ p Value group $1 v$ group 2} \\
\hline & Preop & Postop & p Value & Preop & Postop & p Value & Preop & Postop \\
\hline Heart rate (beats/min) & $68(2.7)$ & $81(2.4)$ & 0.001 & 75 (3.7) & $93(5.5)$ & 0.044 & 0.182 & 0.030 \\
\hline $\mathrm{SBP}(\mathrm{mm} \mathrm{Hg})$ & $115(3.0)$ & $117(2.8)$ & 0.607 & $128(8.3)$ & $117(8.1)$ & 0.001 & 0.076 & 1 \\
\hline $\mathrm{DBP}(\mathrm{mm} \mathrm{Hg})$ & $66(2.4)$ & 64 (1.7) & 0.411 & $77(3.3)$ & $67(3.8)$ & 0.023 & 0.024 & 0.416 \\
\hline $\mathrm{MBP}(\mathrm{mm} \mathrm{Hg})$ & 84 (2.3) & 80 (1.7) & 0.057 & $97(4.3)$ & $85(5.6)$ & 0.005 & 0.010 & 0.259 \\
\hline $\mathrm{RPP}$ (beats/min $\times \mathrm{mm} \mathrm{Hg}$ ) & $7812(396)$ & $9593(424)$ & 0.001 & 9677 (1117) & $10839(900)$ & 0.19 & 0.06 & 0.17 \\
\hline Cardiac output (I/min) & $4.0(0.32)$ & $5.1(0.35)$ & 0.005 & $4.8(1.13)$ & $4.8(0.94)$ & 0.928 & 0.353 & 0.712 \\
\hline PCWP $(\mathrm{mm} \mathrm{Hg})$ & $9(1.0)$ & $10(0.8)$ & 0.097 & $9(0.6)$ & $9(0.8)$ & 1.000 & 1.000 & 0.495 \\
\hline Right atrial pressure $(\mathrm{mm} \mathrm{Hg})$ & $6(0.6)$ & $7(0.6)$ & 0.004 & $5(1.3)$ & $6(1.0)$ & 0.374 & 0.437 & 0.403 \\
\hline PVR (dynes $\times \mathrm{cm} / \mathrm{s}^{-5}$ ) & $1672(136)$ & $1239(66)$ & 0.005 & $1734(327)$ & $1214(113)$ & 0.230 & 0.837 & 0.849 \\
\hline
\end{tabular}

Data are mean (SEM).

DBP, diastolic blood pressure; MBP, mean blood pressure; PCWP, pulmonary capillary wedge pressure; Postop, postoperative data; Preop, preoperative data; PVR, peripheral vascular resistance; RPP, rate-pressure product; SBP, systolic blood pressure. 


\begin{tabular}{|c|c|c|c|c|}
\hline & \multicolumn{2}{|l|}{ Preop } & \multicolumn{2}{|l|}{ Postop } \\
\hline & Measurement 1 & Measurement 2 & Measurement 1 & Measurement 2 \\
\hline \multicolumn{5}{|c|}{ Myocardial blood flow (ml/min) } \\
\hline Group 1 & $59(6.7)$ & $57(6.9)$ & $130(14.0)^{* *}$ & $128(13.2)^{* *}$ \\
\hline Group 2 & $111(20.7)$ & $114(22.2)$ & $106(19.5)$ & $104(20.2)$ \\
\hline $\mathrm{p}$ Value & 0.004 & 0.003 & 0.358 & 0.352 \\
\hline \multicolumn{5}{|c|}{ Myocardial oxygen consumption (ml/min) } \\
\hline Group 1 & $5.9(0.75)$ & $5.6(0.74)$ & $9.7(1.03)^{*}$ & $9.6(0.91)^{*}$ \\
\hline Group 2 & $9.3(1.45)$ & $10.0(2.17)$ & $8.2(1.67)$ & $7.8(1.80)$ \\
\hline $\mathrm{p}$ Value & 0.042 & 0.019 & 0.434 & 0.359 \\
\hline \multicolumn{5}{|c|}{ Myocardial oxygen extraction (\%) } \\
\hline Group 1 & $57(2.6)$ & $56(2.5)$ & $62(2.6)$ & $61(2.5)$ \\
\hline Group 2 & $57(3.6)$ & $59(4.6)$ & $59(2.9)$ & $55(4.4)$ \\
\hline $\mathrm{p}$ Value & 0.958 & 0.527 & 0.489 & 0.273 \\
\hline
\end{tabular}

\section{Blood flow reduction in viable asynergic regions}

Findings of $\mathrm{MBF}$ in viable segments with chronically impaired contractile function conflict in the literature. Reversible left ventricular dysfunction has been alternatively regarded as an adaptive myocardial response to chronically reduced blood flow ${ }^{16}$ and as a consequence of repetitive or cumulative stunning with or without an underlying reduction of baseline blood flow. ${ }^{47}$ The definition of the pathophysiological background underlying the interaction between stunning and hibernation is a debated, still unsolved issue. This dichotomous interpretation essentially comes from positron emission tomography studies where no ${ }^{2418}$ or only slightly $(20-30 \%)^{19}$ reduced blood flow was found in hibernating myocardium with the use of different tracers. Challenging these findings are studies carried out in our laboratory showing that resting $\mathrm{MBF}$ is reduced in the majority of myocardial segments in patients with isolated LAD or circumflex coronary artery stenosis, regardless of the presence or absence of asynergies. ${ }^{20}$ On the other hand, a true reduction in resting MBF has been reported in patients with chronic contractile dysfunction and tissue viability, ${ }^{21}$ in patients with severe postischaemic dysfunction undergoing heart transplantation, ${ }^{22}$ and in patients with stable angina, isolated severe LAD stenosis, and mild contractile impairment undergoing percutaneous transluminal coronary angioplasty. ${ }^{23}$

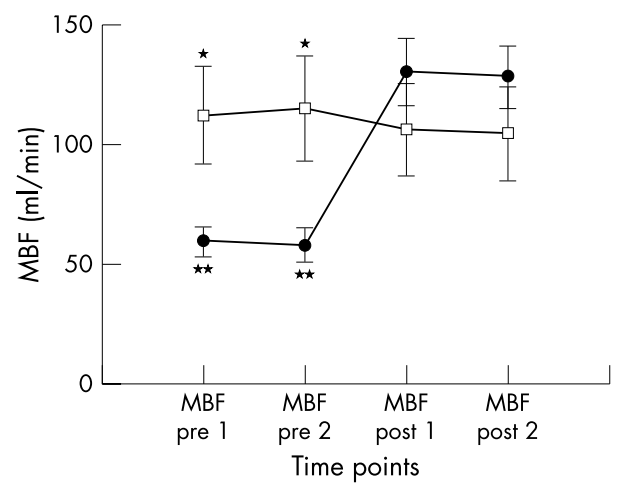

Figure $2 \mathrm{MBF}$ determination in group 1 (circles) and group 2 (squares) patients measured by GCV thermodilution technique. MBF post 1, first postoperative determination; MBF post 2, second postoperative determination; MBF pre 1, first preoperative determination; $M B F$ pre 2, second preoperative determination. ${ }^{*} \mathrm{p}<0.01$ group $1 v$ group 2 before revascularisation; ${ }^{* *} p<0.001$ group $1 \mathrm{MBF}$ before $v$ after revascularisation.
However, the acute setting addressed in this study allowed us to assess simultaneously MBF and metabolism before and after revascularisation and to show that immediate functional recovery is associated with restoration of blood flow and improvement in oxidative metabolism.

\section{Functional recovery after revascularisation}

Similar to initial observations in humans ${ }^{24} 25$ and to findings in animal models, ${ }^{26}$ in our study we found recovery of function soon after revascularisation. Conversely, subacute or chronic recovery lasting from seven days up to several months was reported in experimental ${ }^{27-29}$ as well as in clinical $^{30}{ }^{31}$ studies. Several methodological aspects, including characteristics of the studied population, experimental design, and parameters used to assess viability and functional recovery, have to be considered to explain these differences. In particular, preoperative ejection fraction was just slightly compromised in our patients; moreover, none of them had acute myocardial infarction, and tissue viability was found in 71 of 78 (91\%) LAD related dysfunctional segments by imaging techniques. These characteristics identify a selected model of hibernation with a high proportion of preserved
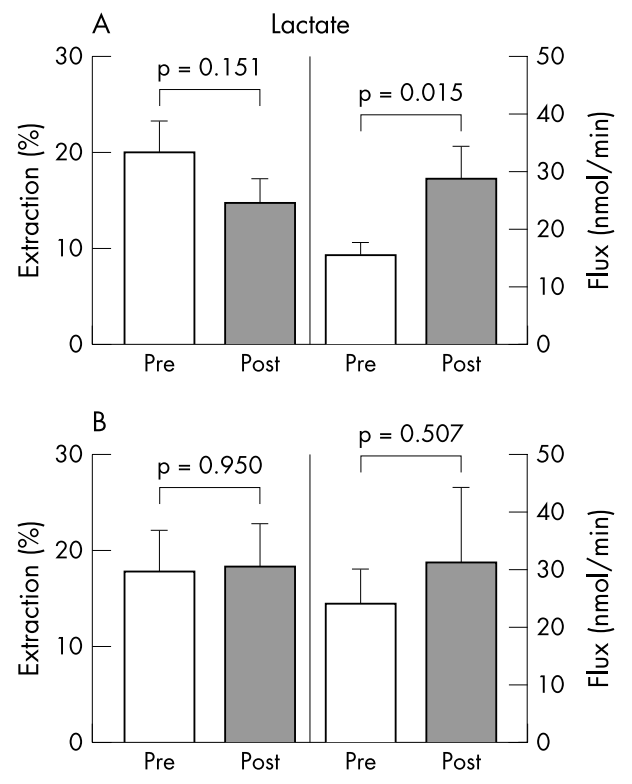

Figure 3 Myocardial extractions and transmyocardial fluxes of lactate in (A) group 1 and (B) group 2 patients. 

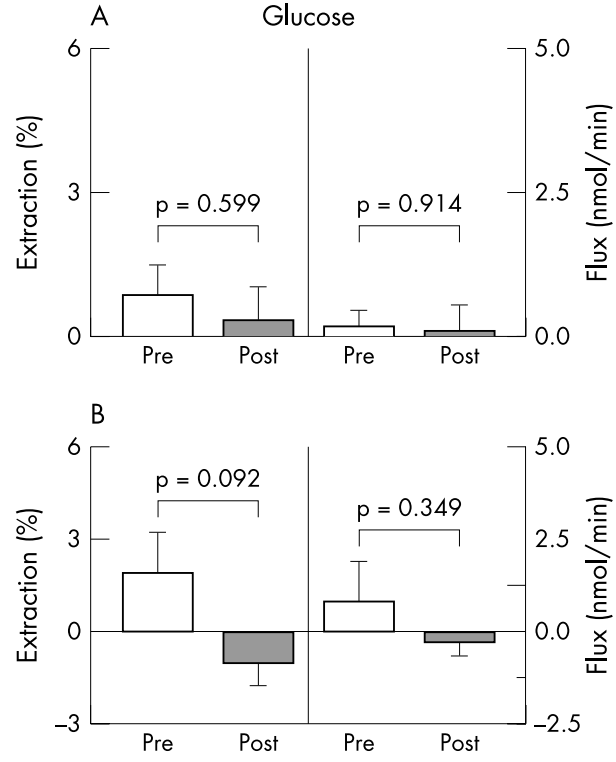

Figure 4 Myocardial extractions and transmyocardial fluxes of glucose in (A) group 1 and (B) group 2 patients.

myocytes $^{32}{ }^{33}$ where the degree of structural alterations is not likely to prevent early functional recovery. Conversely, delayed recovery of function is expected in the case of severe morphological changes. ${ }^{51-33}$ In addition, evidence of oxidative metabolism in segments with reduced blood flow is consistent with the hypothesis of a true transmural decline in flow in otherwise viable tissue rather than pseudoreduction secondary to a mixture of scarred and normal tissue. On the other hand, the presence of a transmurally extended patchy component of scarred myocardium may interfere with echocardiographic detection of contractile reserve. ${ }^{34}$

The duration of hibernation is also relevant in determining the rate of functional recovery. ${ }^{27}$ Although we have no definite information on this issue, LV dysfunction had been detected at least one month before surgery by protocol in our patients; moreover, onset of clinical symptoms lasted no longer than one year. Finally, rapid recovery has been suggested to reflect only transient improvement in function due to afterload reduction or catecholamine stimulation. ${ }^{24} 25$ However, improved contractile function was still detectable in our study population three months after revascularisation when the haemodynamic changes induced by surgery were no longer present.

The experimental setting of this study-that is, surgical revascularisation in patients with sustained resting hypoperfusion-may have magnified the potential role of ischaemic preconditioning in providing metabolic protection against reperfusion injury after weaning from bypass and consequent fast functional recovery. Therefore, the results of the present study, in a group of patients with stable angina and a high percentage of viable myocardium in dysfunctional areas, support the hypothesis that resting MBF is greatly reduced in this clinical setting. In fact, MBF was immediately restored after revascularisation to values similar to those of patients with normal function, whereas blood flow in the LAD related territories of the latter group did not change after CABG surgery.

\section{$M B F$, oxygen utilisation, and metabolism}

The increase in MBF in viable myocardium soon after revascularisation was paralleled by an increase in myocardial oxygen consumption in the same territories, whereas there was a very slight decrease in oxygen extraction. This finding
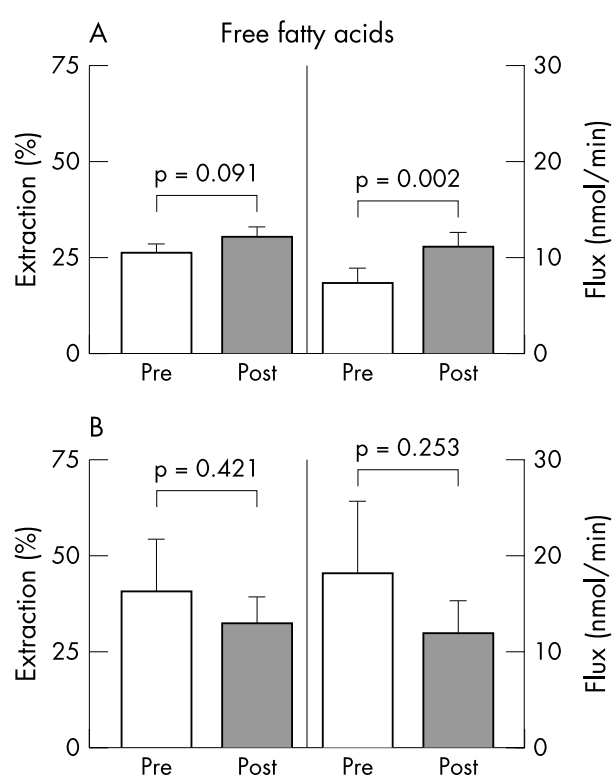

Figure 5 Myocardial extractions and transmyocardial fluxes of free fatty acids in (A) group 1 and (B) group 2 patients.

indicates that the increase in blood flow in the immediate postoperative period supplies viable areas, allowing a substantial increase of their preoperative aerobic metabolism. The possibility that increases in MBF may be caused by a hyperaemic response related to aortic cross clamping and reperfusion can be ruled out because the pre-revascularisation and post-revascularisation oxygen extraction rates were similar in both groups of patients and the repeated postrevascularisation measurements of MBF, myocardial oxygen consumption, and oxygen extraction were stable over time.

Our study also shows that the previously asynergic segments recovered their contractility at the same time as MBF and myocardial oxygen consumption increased, and that this occurred under haemodynamic conditions that were as similar as possible to those existing before surgery. The confirmation of this immediate recovery of contractility by follow up echocardiograms with no further improvement suggests that, at least in our patient population, the mechanisms underlying myocardial hibernation should be ascribed to an adaptive metabolic condition rather than to cell dedifferentiation ${ }^{4}$ or cell degeneration. ${ }^{5}$ In fact, these mechanisms would require some time before restoration of contractile function.

Our data suggest that hibernating territories maintain an oxidative metabolic pattern before and after revascularisation; in fact, the glucose extraction ratio and uptake did not

Table 4 Wall motion score index of LAD related asynergic segments before and after surgical myocardial revascularisation in group 1 patients

\begin{tabular}{ll}
\hline Echocardiographic method & $\begin{array}{l}\text { Wall motion } \\
\text { score index }\end{array}$ \\
\hline $\begin{array}{l}\text { Preoperative transthoracic } \\
\text { Dobutamine stress }\end{array}$ & $2.8(0.74)$ \\
Transoesophageal & $1.6(1.01)^{*}$ \\
$\quad$ Preoperative & $2.6(0.85)$ \\
Postoperative & $1.5(0.98)^{*}$ \\
8 days' follow up & $1.6(1.00)^{*}$ \\
3 months' follow up & $1.6(1.05)^{*}$ \\
\hline Data are mean (SEM). & \\
*p $<0.01$ v preoperative transthoracic and preoperative \\
transoesophageal echocardiography.
\end{tabular}




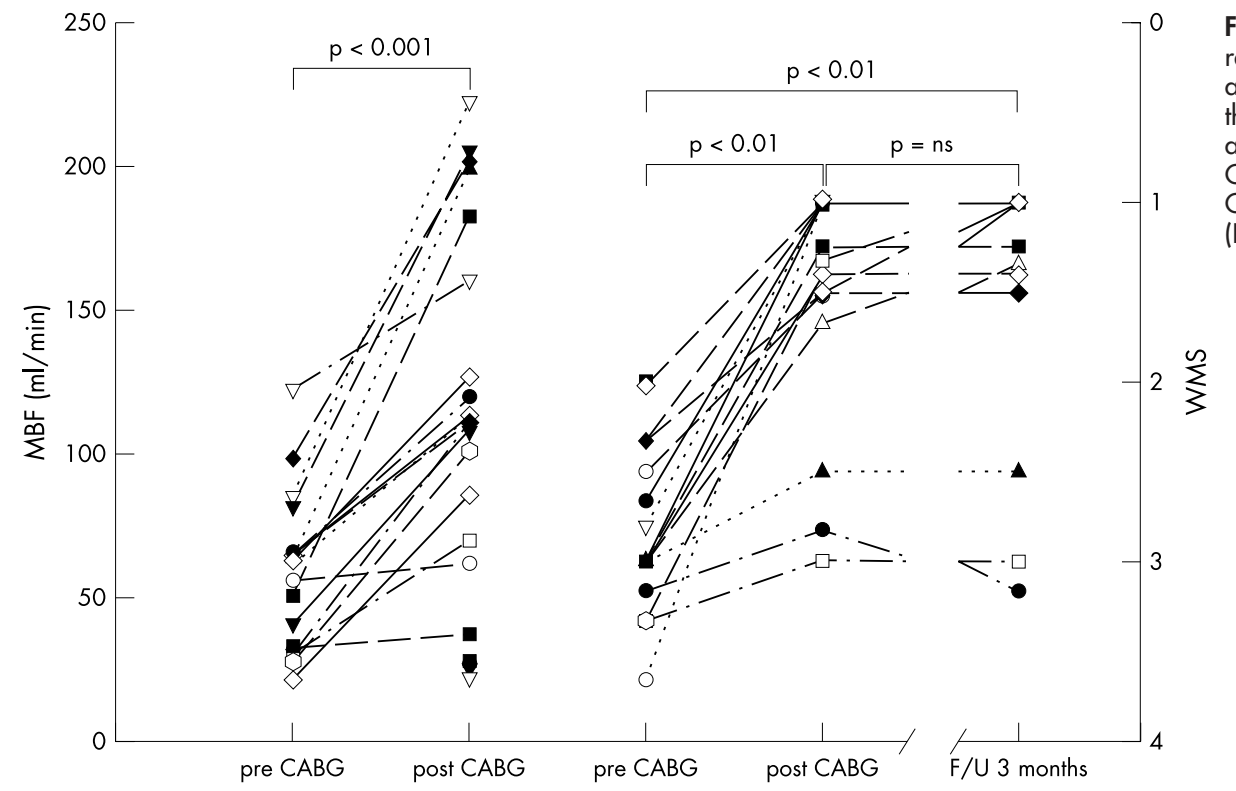

Figure 6 Individual behaviour of (left) rate-pressure product corrected $\mathrm{MBF}$ and (right) wall motion score (WMS) of the left anterior descending coronary artery related territories before (pre $C A B G$ ) and after revascularisation post $\mathrm{CABG})$, and at three months' follow up (F/U). ns, not significant.

change in group 1 patients, whereas lactate and free fatty acid uptakes increased significantly after revascularisation. The increase in transmyocardial flow and consequent uptake of both lactate and free fatty acids in group 1, together with the lack of significant differences in utilisation of these substrates in group 2, adds further strength to the hypothesis that the immediate post-revascularisation increase in the MBF of hibernating LAD areas is related to a prompt increase in substrate oxidative utilisation.

\section{Study limitations}

The limitations of this study are that LAD related dysfunctional territories may not have been homogeneous, with normal, necrotic, and hibernating myocardium, and so our evaluation of their blood flow and metabolism may reflect different pathophysiological situations simultaneously expressed in different LAD territories. However, we selected patients with evidence of myocardial viability in most of the asynergic segments at ${ }^{201} \mathrm{Tl}-\mathrm{SPECT}$ or dobutamine stress echocardiography ( $>90 \%$ of viable segments).

Another possible criticism is that we used the thermodilution technique to assess MBF in LAD territories, the accuracy of which may be affected by heart motion or interventions leading to large changes in heart size. However, the reflux of right atrial blood into the coronary sinus (which may lead to a spurious overestimate) can be ruled out on the basis of the visually checked advanced positioning of the catheter tip in the great cardiac vein.

Established techniques for measuring $\mathrm{MBF}$ are positron emission tomography, the microsphere technique, and intracoronary Doppler flow wires, which provide accurate estimates of regional myocardial perfusion. However, in our surgical experimental setting, the thermodilution modality was the only suitable, safe, and reliable technique to calculate resting MBF.

Lastly, the restricted range of our time points (30 minutes after weaning from cardiopulmonary bypass) does not permit the extension of these metabolic observations to the subsequent postsurgical course.

\section{Clinical implications}

Our findings indicate that, when a high degree of viability in asynergic myocardial areas can be documented preoperatively by means of imaging techniques in patients with postischaemic dysfunctional myocardium, revascularisation is followed by a major increase in coronary blood flow and a prompt recovery of contractility. The mechanism underlying myocardial hibernation in this cohort of patients, with stable angina pectoris and relatively recent onset of symptoms (less than one year), can be ascribed to an adaptive mechanism to preserve myocyte integrity in the setting of chronic coronary flow restriction, which can be immediately improved by effective revascularisation. We are aware that our conclusions may apparently conflict with the emerging interpretation of hibernating myocardium as a result of chronic stunning. However, the peculiar characteristics of our experimental setting-that is, functional assessment immediately after blood flow restoration-provide a logical explanation for the disagreement with part of the previous literature.

The use of non-invasive tests aimed at evaluating the lack of pathological progression in hibernating myocardium may identify patients in whom contractility most probably will improve immediately after surgical revascularisation. This insight appears to be helpful in clinical decision making and management of those patients during surgical procedures.

\section{ACKNOWLEDGEMENTS}

This study was supported in part by a grant from the Ministry of Health (ICS030.6/RF96.309), Italy. Dr Marina Parolini's contribution of data analysis was greatly appreciated.

\section{Authors' affiliations}

F Alamanni, A Parolari, E Doria, M Pepi, E Sisillo, M Naliato, P Biglioli, Centro Cardiologico Monzino, IRCCS, Department of Cardiac Surgery and Cardiology, University of Milan, Milan, Italy

J Campolo, R Bigi, O Parodi, CNR Clinical Physiology Institute, Section of Milan, Cardiothoracovascular Department, Niguarda Hospital, Milan, Italy

A Repossini, F Bortone, Department of Cardiology, Humanitas Gavazzeni, Bergamo, Italy

\section{REFERENCES}

1 Rahimtoola SH. Clinical aspects of hibernating myocardium. J Mol Cell Cardiol 1996;28:2397-401.

2 Gerber BL, Vanoverschelde JL, Bol A, et al. Myocardial blood flow, glucose uptake, and recruitment of inotropic reserve in chronic left ventricular ischemic dysfunction. Implications for the pathophysiology of chronic myocardial hibernation. Circulation 1996:94:651-9.

3 Maki $M$, Luotolahti $M$, Nuutila $P$, et al. Glucose uptake in the chronically dysfunctional but viable myocardium. Circulation 1996;93:1658-66.

4 Vanoverschelde JL, Wiins W, Depre C, et al. Mechanisms of chronic regional postischemic dysfunction in humans. New insights from the study of 
noninfarcted collateral-dependent myocardium. Circulation 1993;87:1513-23.

5 Elsasser A, Schlepper M, Klovekorn WP, et al. Hibernating myocardium: an incomplete adaptation to ischemia. Circulation 1997;96:2920-31.

6 La Canna G, Alfieri O, Giubbini R, et al. Echocardiography during infusion of dobutamine for identification of reversible dysfunction in patients with chronic coronary artery disease. J Am Coll Cardiol 1994;23:617-26.

7 Bharati S, Maes A, Borgers $M$, et al. Only hibernating myocardium shows early recovery after coronary revascularization. Circulation 1996;94:308-15.

8 Carr KW, Engler RL, Forsyte JR, et al. Measurements of left ventricular ejection fraction by mechanical cross-sectional echocardiography. Circulation 1979;59:1196-206

9 Afridi I, Kleinman NS, Raizner AE, et al. Dobutamine echocardiography in myocardial hibernation: optimal dose and accuracy in predicting recovery of ventricular function after coronary angioplasty. Circulation 1995.91:990-8.

10 Parolari A, Guarnieri D, Alamanni F, et al. Platelet function and anesthetics in cardiac surgery: an in vitro and ex vivo study. Anesth Analg 1999;89:26-31.

11 Baim DS, Rothman MT, Harrison DC. Improved catheter for regional coronary sinus blood flow and metabolic studies. Am J Cardiol 1980;46:997-1000.

12 Marinho NVS, Keogh BE, Costa DC, et al. Pathophysiology of chronic left ventricular dysfunction. New insights from the measurement of absolute myocardial blood flow and glucose utilization. Circulation 1996;93:737-44.

13 Noll F. I-(+)-Lactate. In: Bergmeyer HU, ed. Methods of enzymatic analysis, 3rd ed. Weinheim: Verlag Chemie, 1981:582-8.

14 Shimizu $S$, Inove $K$, Tani $Y$, et al. Enzymatic microdetermination of serum free fatty acids. Anal Biochem 1979;98:341-5.

15 Bland JM, Altman DG. Statistical methods for assessing agreement between two methods of clinical measurement. Lancet 1986;i:307-10.

16 Rahimtoola SH. Hibernating myocardium has reduced blood flow at rest that increases with low-dose dobutamine. Circulation 1996;94:3055-61.

17 Camici PG, Wiins W, Borgers M, et al. Pathophysiological mechanisms of chronic reversible left ventricular dysfunction due to coronary artery disease (hibernating myocardium). Circulation 1997:96:3205-14.

18 Conversano A, Walsh JF, Geltman EM, et al. Delineation of myocardial stunning and hibernation by positron emission tomography in advanced coronary artery disease. Am Heart J 1996;131:440-50.

19 Grandin C, Wijns W, Melin JA, et al. Delineation of myocardial viability with PET. J Nucl Med 1995;36:1543-52.

20 Sambuceti G, Marzullo P, Giorgetti A, et al. Global alteration in perfusion response to increasing oxygen consumption in patients with single-vessel coronary artery disease. Circulation 1994;90:1696-705.

21 Tawakol A, Skopicki HA, Abraham SA, et al. Evidence of reduced resting blood flow in viable myocardial regions with chronic asynergy. J Am Coll Cardiol 2000;36:2146-53
22 Parodi O, De Maria R, Oltrona L, et al. Myocardial blood flow distribution in patients with ischemic heart disease or dilated cardiomyopathy undergoing heart transplantation. Circulation 1993;88:509-22.

23 Parodi O, Sambuceti G, Roghi A, et al. Residual coronary reserve despite decreased resting blood flow in patients with critical coronary lesion: a study by technetium- $99 \mathrm{~m}$ human albumin microsphere myocardial scintigraphy. Circulation 1993;87:330-44

24 Topol EJ, Weiss JL, Guzman PA, et al. Immediate improvement of dysfunctional myocardial segments affer coronary revascularization: detection by transesophageal echocardiography. J Am Coll Cardiol 1984;4: 1 123-34.

25 Cohen M, Charney R, Hershman R, et al. Reversal of chronic myocardial dysfunction after transluminal coronary angioplasty. J Am Coll Cardiol 1988; 12:1193-8.

26 Ferrari R, Cargnoni A, Bernocchi $P$, et al. Metabolic adaptation during a sequence of no-flow and low-flow ischemia: a possible trigger for hibernation. Circulation 1996:94:2587-96.

27 Matsusaki M, Gallagher KP, Kemper WS, et al. Sustained regional dysfunction produced by prolonged coronary stenosis: gradual recovery after reperfusion. Circulation 1983;68:170-82.

28 Chen C, Chen L, Fallon JT, et al. Functional and structural alteration with 24hour myocardial hibernation and recovery after reperfusion: a pig model of myocardial hibernation. Circulation 1996;94:507-16.

29 Perrone-Filardi P, Pace L, Rastaro M, et al. Dobutamine echocardiography predicts improvement of hypoperfused dysfunctional myocardium after revascularization in patients with coronary artery disease. Circulation 1995;91:2256-65.

30 Bax JJ, Visser FC, Poldermans D, et al. Time course of functional recovery of stunned and hibernating segments after surgical revascularization. Circulation 1999:100:490-6.

31 Depré C, Vanoverschelde JL, Melin JA, et al. Structural and metabolic correlates of the reversibility of chronic left ventricular ischemic dysfunction in humans. Am J Physiol 1995;268:H1265-75.

32 Nagueh S, Mikati I, Weilbaecher D, et al. Relation of the contractile reserve of hibernating myocardium to myocardial structures in humans. Circulation $2001 ; 104$ (suppl):1314-8.

33 Gunning MG, Kaprielan RR, Pepper J, et al. The histology of viable and hibernating myocardium in relation to imaging characteristics. J Am Coll Cardiol 2002;39:428-35.

34 Liebermann AN, Weiss JL, Judgutt BI, et al. Two-dimensional echocardiography and infarct size: relationship of regional wall motion and thickening to the extent of myocardial infarction in the dog. Circulation $1981 ; 63: 739-46$

\section{IMAGES IN CARDIOLOGY}

\section{Delayed presentation of right ventricular bullet embolus}

A 68 year old man presented in 1992 with syncope related to complete heart block. A chest radiograph showed a small calibre bullet that appeared to be within the heart. A permanent pacemaker was implanted.

He had fought on the Russian front during the second world war, where he was wounded on five occasions. On the last of these he sustained a bullet wound to the left side of his neck below the angle of the jaw that healed, although the bullet was not recovered

Chest radiographs recorded in 2003 (59 years after he was wounded) show the pacing electrode $\left({ }^{*}\right)$ and the bullet, which echocardiography showed was within the cavity of the right ventricle.

N Bett
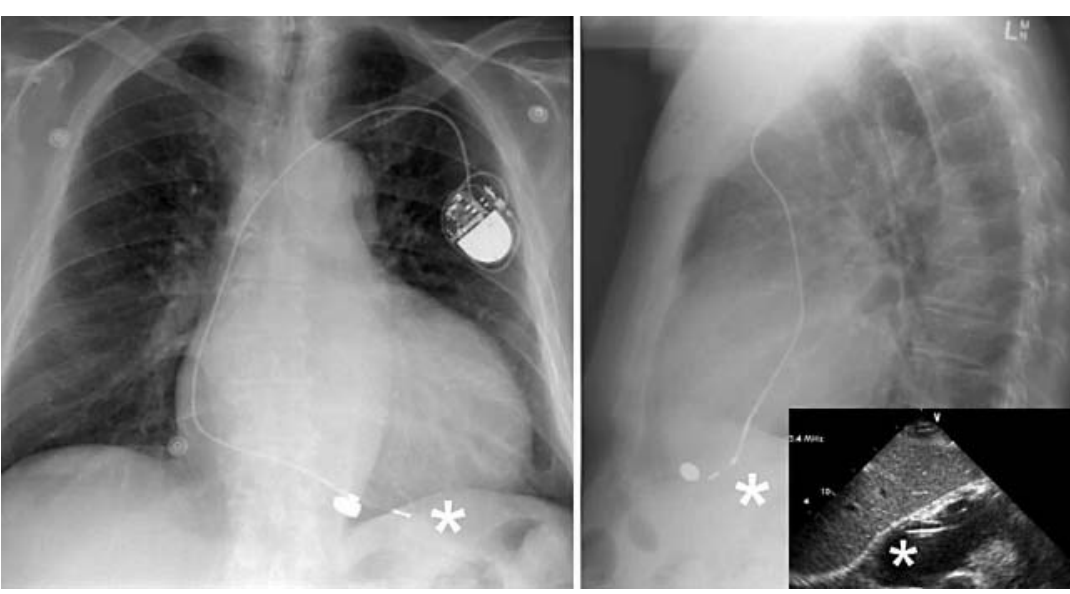